\title{
The role of primary tumor SUVmax in the diagnosis of invasion depth: a step toward clinical T2NO esophageal cancer
}

\author{
Dong Lin ${ }^{1 \#}$, Guobing Liu ${ }^{2 \#}$, Dongxian Jiang ${ }^{3 \#}$, Yangli Yu ${ }^{4}$, Hao Wang ${ }^{1}$, Hongcheng Shi ${ }^{2}$, Lijie Tan ${ }^{1}$ \\ ${ }^{1}$ Department of Thoracic Surgery, Zhongshan Hospital Fudan University, Shanghai, China; ${ }^{2}$ Department of Nuclear Medicine, Zhongshan Hospital \\ Fudan University, Shanghai, China; ${ }^{3}$ Department of Pathology, Zhongshan Hospital Fudan University, Shanghai, China; ${ }^{4}$ Department of Radiology, \\ Zhongshan Hospital Fudan University, Shanghai, China \\ Contributions: (I) Conception and design: L Tan, H Shi; (II) Administrative support: L Tan, H Shi; (III) Provision of study materials or patients: \\ H Wang; (IV) Collection and assembly of data: D Lin, G Liu, D Jiang, Y Yu; (V) Data analysis and interpretation: D Lin, G Liu, D Jiang; (VI) \\ Manuscript writing: All authors; (VII) Final approval of manuscript: All authors. \\ \#These authors contributed equally to this work. \\ Correspondence to: Lijie Tan, MD, FACS. Department of Thoracic Surgery, Zhongshan Hospital Fudan University, 180 Fenglin Rd., Shanghai 200032 , \\ China. Email: tan.lijie@zs-hospital.sh.cn.
}

Background: The controversy regarding optimal clinical T2N0 esophageal cancer treatment ultimately stems from the clinical staging modalities' inaccuracy. Because most inaccuracies lie in clinical T2 to pathological T1, it is vital to discriminate whether the muscularis propria is invaded.

Methods: We investigated the association between the primary tumor maximal standard uptake value (SUVmax), and the pathological features and overall survival. We attempted to construct a discriminative model through logistic regression analysis.

Results: A total of $140 \mathrm{cN} 0$ esophageal squamous cell carcinoma (ESCC) patients were enrolled. Primary tumor SUVmax differed significantly in paired pathological $\mathrm{T}$ categories $(\mathrm{P}<0.05)$, but not pT2 vs. pT3 $(\mathrm{P}=0.648)$. Age ( $\leq 65 v s$. $>65)$, biopsy differentiation grades (well or moderately $v s$. poorly $v s$. unknown), and primary tumor SUVmax (continuous) were independent risk factors for invasion depth. Subsequently, the age categories, the biopsy differentiation grade categories, and the primary tumor SUVmax categories $(\leq 7.4$ $v s$. 7.4) were included in the logistic regression analysis to construct a discriminative model, showing a good performance in discriminating pT2-3 vs. pT1 in terms of accuracy $87.1 \%$, sensitivity $93.6 \%$, specificity $73.9 \%$, and area under the curve (AUC) 0.887 [95\% confidence interval (CI): 0.822 to 0.951 ]. Of these factors, biopsy differentiation grades and primary tumor SUVmax showed significant differences in overall survival $(\mathrm{P}<0.05)$, while the age categories did not.

Conclusions: The novel baseline model comprised of age, biopsy differentiation grades, and primary tumor SUVmax provide much discriminative performance in determining whether the muscularis propria is invaded. Further studies are necessary to validate the findings and guide clinical practice for cT2N0 esophageal cancer.

Keywords: Esophageal cancer; invasion depth; positron emission tomography with computed tomography (PETCT); maximal standard uptake value (SUVmax)

Submitted Jun 02, 2020. Accepted for publication Oct 30, 2020.

doi: $10.21037 / \mathrm{atm}-20-4430$

View this article at: http://dx.doi.org/10.21037/atm-20-4430

^ ORCID: 0000-0002-6098-0632. 


\section{Introduction}

Esophageal cancer is one of the most common malignancies worldwide, with a dismal prognosis (1). Histologically, esophageal cancer includes two major subtypes: esophageal adenocarcinoma (EAC) and esophageal squamous cell carcinoma (ESCC) (2). In recent decades, much attention has been paid towards improving patients' long-term survival with esophageal cancer. Nowadays, induction therapy (chemotherapy or chemoradiotherapy), followed by surgery, can provide a survival benefit for locally advanced esophageal cancer (3-6). Local therapy (endoscopic or surgical resection) is usually applied for early-stage esophageal cancer, but this remains the subject of much debate.

This debate is derived from the inaccuracy of esophageal cancer's clinical staging, which is particularly evident in clinical T2N0 (cT2N0). Crabtree et al. found that 34\%, $25.3 \%$, and $0.6 \%$ of cT2N0 were pathological T0-1 (pT0-1), pT3, and pT4, respectively (7). The Esophageal Cancer Study Group found that $44.5 \%, 31 \%$, and $1 \%$ of cT2N0 were pT0-1, pT3, and pT4, respectively (8). Other groups have reported that more than $50 \%$ of cT2 esophageal cancers were pT1 after surgery, even when staged by experienced clinicians $(9,10)$. Consequently, there is much debate surrounding the optimal treatment for $\mathrm{cT} 2 \mathrm{~N} 0$ esophageal cancer, namely, between primary surgery or induction therapy (11-13).

The current pathological $\mathrm{T}$ staging is based on invasion depth. Thus, an accurate clinical $\mathrm{T}$ staging demands a high resolution of the esophagus (14), especially for flat tumors and diffused infiltration (15). The fact that most migration of the cT2 category lies in cT2 to pT1 indicates that the common modalities cannot accurately discriminate whether or not the muscularis propria is invaded. Thus, one natural question is whether the metabolic activity may play a role in this process.

${ }^{18} \mathrm{~F}$-fluorodeoxyglucose $\left({ }^{18} \mathrm{~F}\right.$-FDG $)$ positron emission tomography (PET) with computed tomography (CT) is included as part of the initial workup (3-5), but it is usually used to detect distant metastasis (16). Recent studies have found that the maximal standard uptake value (SUVmax) of the primary tumors was associated with pathological features (17-26). We, therefore, hypothesized that metabolic activity would determine whether or not the muscularis propria was invaded. We investigated the potential association between the primary tumor SUVmax and pathological features and overall survival. Furthermore, we attempted to construct certain models to discriminate invasion depth. We present the following article in accordance with the STARD reporting checklist (available at http://dx.doi.org/10.21037/ atm-20-4430).

\section{Methods}

\section{Patients}

From January 2015 to December 2017, 234 consecutive patients underwent baseline ${ }^{18} \mathrm{~F}$-FDG PET/CT, followed by primary esophagectomy in Zhongshan Hospital Fudan University. Of these patients, 67 were clinically staged as $\mathrm{cN}+$, while 2 had received endoscopic dissection (ESD) before ${ }^{18} \mathrm{~F}$-FDG PET/CT, and 25 were histologically confirmed as not ESCC. Eventually, 140 patients were enrolled in this retrospective study. This study had been approved by the Institutional Review Board of Zhongshan Hospital Fudan University (HGBB-202006001) and was conducted in accordance with the Declaration of Helsinki (as revised in 2013). The informed consent was waived as the nature of retrospective studies.

As the reference standard, the pathological staging was based on the TNM Staging System ( $8^{\text {th }}$ edition, 2017) (14). Tumors that invaded the lamina propria or muscularis mucosae, the submucosa, the muscularis propria, the adventitia, and the adjacent structures were defined as $\mathrm{pT} 1 \mathrm{a}$, pT1b, pT2, pT3, and pT4, respectively. All of the patients had discernible $\mathrm{p} T$ categories in this study.

\section{Follow-up}

Patients were asked to receive physical examination, tumor markers testing, thoracic CT, and cervical and abdominal ultrasonography in the outpatient clinic. For patients with particular signs or symptoms, additional examinations were conducted. A combination of clinical service records and phone calls were used to determine each patient's status as of March 2020. In this study, overall survival was defined as the interval between the date of esophagectomy and the date of death or the last follow-up date.

\section{PET/CT protocol and interpretation}

According to the routine protocol of ${ }^{18} \mathrm{~F}$-FDG PET-CT at our institution, the patients fasted for at least 6 hours, and the serum glucose levels were required to be lower than $11.0 \mathrm{mmol} / \mathrm{L}$ before imaging. ${ }^{18} \mathrm{~F}$-FDG PET/CT scans were performed on a hybrid GE Discovery VCT 64 PET/ CT scanner (General Electric, Milwaukee, WI, USA) from 


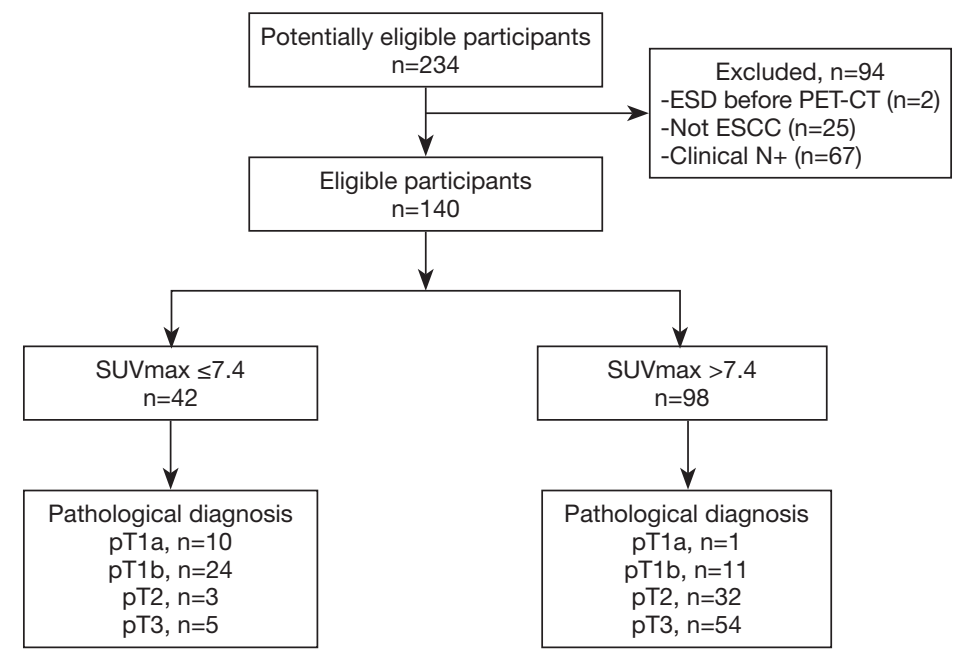

Figure 1 The flowchart of the enrollment and the discriminating outcomes of SUVmax (categorized). SUVmax, maximal standard uptake value; ESD, endoscopic dissection; PET-CT, positron emission tomography with computed tomography; ESCC, esophageal squamous cell carcinoma.

the proximal thigh to the skull base. Metabolic images were obtained approximately 60 minutes after intravenous administration of 3.7 to $5.6 \mathrm{MBq}$ of FDG per kilogram of body weight. PET images were acquired for 2 minutes per bed position. The CT scanning was performed on the same scanner without contrast administration $(200 \mathrm{~mA}$, $120 \mathrm{kV}$, matrix $512 \times 512,0.8 \mathrm{~s}$ per rotation). The SUV was normalized to body weight as a determinate index. All PET-CT scans were performed within 1 month before surgery.

\section{Statistical analyses}

Continuous data were presented as mean with standard deviation (SD) or median with interquartile ranges (IQRs). Categorical data were presented as numbers with a proportion (\%). Both parametric and non-parametric tests were used, including the Chi-square test and the KruskalWallis test. The association between baseline characteristics and pathological findings was determined by univariate and multivariate logistic regression analysis. The variables with $\mathrm{P}<0.10$ in the univariate regression were included in the multivariate regression. The independent factors were included in the predictive model. The receiver operator curve (ROC) with Youden's index (27) was used to determine the predictive performance and identify the optimal cutoff of continuous parameters. The area under the curve (AUC) with $95 \%$ confidence interval $(\mathrm{CI})$ was used to measure the discriminating performance. The Kaplan-Meier method was used to generate survival curves, and the log-rank test was used to evaluate survival differences. All patients included in the survival analysis were followed up for at least 3 months after surgery or until death.

Statistical analyses were performed using SPSS version 24 (SPSS Inc., Chicago, IL, USA) and R software version 1 3.5.1 (Packages: survival and survminer). $\mathrm{P}<0.05$ was considered statistically significant.

\section{Results}

\section{Preoperative characteristics of the patients}

As shown in Figure 1 and Table 1, a total of $140 \mathrm{cN} 0$ patients were enrolled in this study, including 25 females and 115 males, with a median age of 65 (IQR: $60.25-71.00$ ). Of the 140 patients, $74(52.9 \%)$ were clinically staged as cT1-2, and $66(47.1 \%)$ were clinically staged as cT3. Based on the endoscopic biopsy, $41(29.3 \%)$ tumors were poorly differentiated, $84(60.0 \%)$ tumors were well or moderately differentiated, while 15 (10.7\%) tumors had no grading information. The median and mean SUVmax were 11.35 (IQR: 6.28-15.68) and 11.37 (SD: 6.10), respectively.

\section{Postoperative characteristics and their association with SUVmax}

For all patients, surgical resection with the pathological 
Table 1 Preoperative characteristics in the $\mathrm{cN} 0$ patients

\begin{tabular}{|c|c|}
\hline Characteristics & $\begin{array}{c}\text { Median (IQR) or } \\
\text { number (percentage) }\end{array}$ \\
\hline \multicolumn{2}{|l|}{ Age } \\
\hline Median (IQR) & $65(60.25-71.00)$ \\
\hline \multicolumn{2}{|l|}{ Sex } \\
\hline Female & $25(17.9)$ \\
\hline Male & $115(82.1)$ \\
\hline \multicolumn{2}{|l|}{ Smoking history } \\
\hline Non-smoking & $93(66.4)$ \\
\hline Smoking & $47(33.6)$ \\
\hline \multicolumn{2}{|l|}{ Drinking history } \\
\hline Non-drinking & $95(67.9)$ \\
\hline Drinking & $45(32.1)$ \\
\hline \multicolumn{2}{|l|}{ Clinical T } \\
\hline cT1-2 & $74(52.9)$ \\
\hline сT3 & $66(47.1)$ \\
\hline \multicolumn{2}{|l|}{ Differentiation of biopsy sample } \\
\hline bG1, 2, x (well, moderately) & $84(60.0)$ \\
\hline bG3 (poorly) & $41(29.3)$ \\
\hline Unknown & $15(10.7)$ \\
\hline \multicolumn{2}{|l|}{ Surgical procedure } \\
\hline MIE & $84(60.0)$ \\
\hline Open & $56(40.0)$ \\
\hline \multicolumn{2}{|l|}{ SUVmax } \\
\hline Median (IQR) & $11.35(6.28-15.68)$ \\
\hline Mean (SD) & $11.37(6.10)$ \\
\hline \multicolumn{2}{|l|}{ Surgery year } \\
\hline 2015 & $26(18.6)$ \\
\hline 2016 & $46(32.8)$ \\
\hline 2017 & $68(48.6)$ \\
\hline
\end{tabular}

IQR, interquartile range; bG, biopsy differentiation grade; MIE, minimally invasive esophagectomy; open, open esophagectomy; SUVmax, maximal standard uptake value; SD, standard deviation.

examination was conducted within 1 month after the completion of PET-CT, and no cancer-related treatment was administered during this interval. Histologically, 11 (7.9\%), 35 (25.0\%), 35 (25.0\%), and $59(42.1 \%)$ tumors

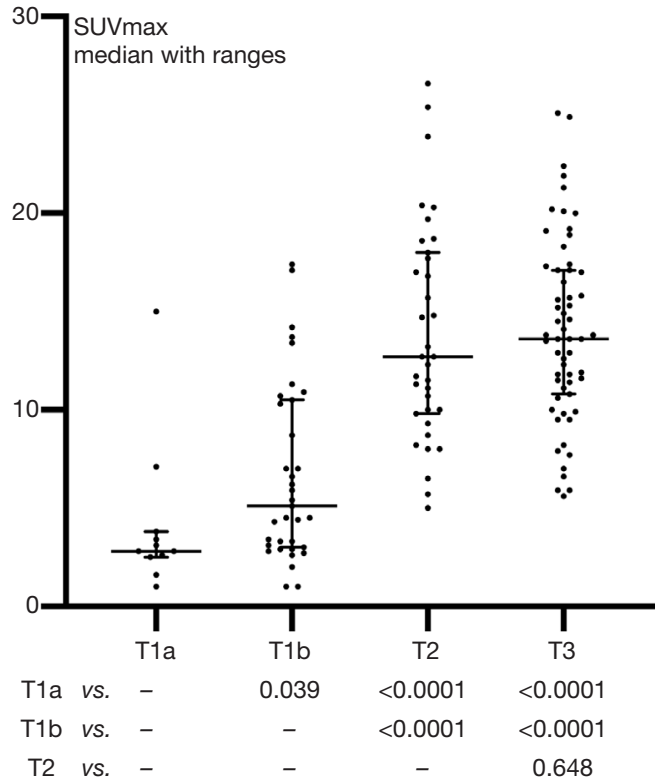

Figure 2 The primary tumor SUVmax in pathological T categories. SUVmax of primary tumors differed significantly in paired pT categories (all $\mathrm{P}<0.05)$ but not pT2 vs. pT3 $(\mathrm{P}=0.648)$. SUVmax, maximal standard uptake value.

were confirmed as pT1a (mucosa), pT1b (submucosa), pT2 (muscularis propria), and pT3 (adventitia), respectively, while $36(25.7 \%)$ were staged as $\mathrm{pN}+$. There was a significant correlation between invasion depth and node metastasis ( $\mathrm{P}=0.017$, Table S1). Ultimately, 110 (78.6\%) were in pathological stage I-II, and $30(21.4 \%)$ were in pathological stage III-IV. Lymphatic or nerve invasion (LNI) was present in 43 (30.7\%) patients.

As shown in Figure 2, the primary tumor SUVmax differed significantly between any paired $\mathrm{p} T$ categories (all $\mathrm{P}<0.05)$, but not between pT2 vs. pT3 ( $\mathrm{P}=0.648)$. As shown in Table 2, although SUVmax also differed significantly between pathological stage categories (pStage I $v s$. II $v s$. IIIIV, $\mathrm{P}<0.0001$ ), we found it was not significantly different in $\mathrm{pN}$ categories ( $\mathrm{pN} 0$ vs. $\mathrm{pN}+, \mathrm{P}=0.187$ ), $\mathrm{pG}$ categories (well or moderately $v s$. poorly, $\mathrm{P}=0.254$ ), and LNI categories (absence $v$ s. presence, $\mathrm{P}=0.062$ ).

\section{Baseline risk factors for invasion depth}

According to the univariate and multivariate logistic regression analysis, age ( $\leq 65 v s .>65)$, biopsy differentiation (well or moderately $v s$. poorly $v s$. unknown), and SUVmax (continuous) were identified as the baseline risk factors for 
Table 2 Postoperative characteristics and the association with SUVmax in the cN0 patients

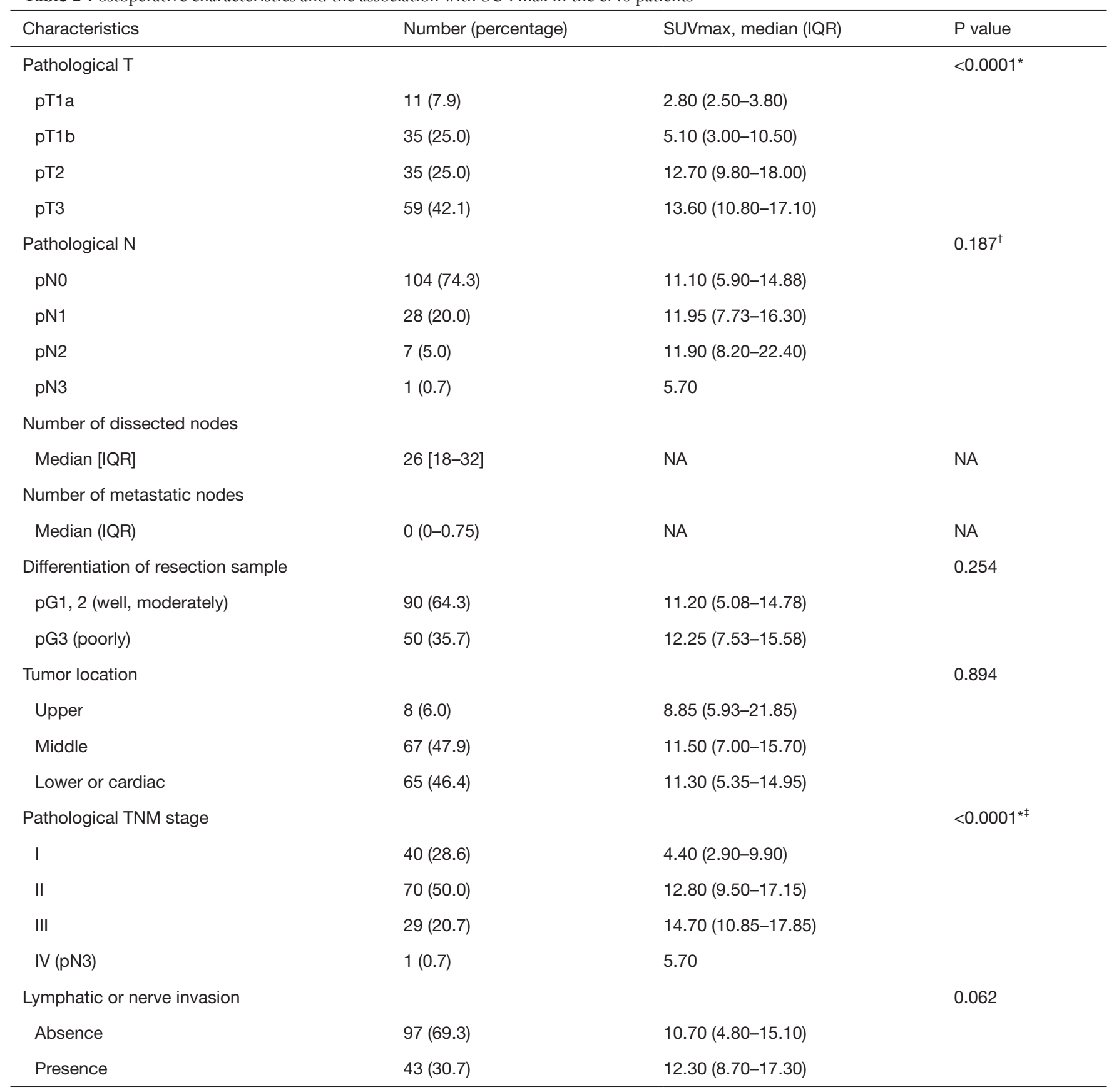

${ }^{*}$, statistically significant; ${ }^{\dagger}$, pN0 vs. pN1-3; ${ }^{\ddagger}$, pathological stage I vs. stage II vs. stage III-IV. SUVmax, maximal standard uptake value; IQR, interquartile range; NA, not applicable.

the invasion of the muscularis propria (Table 3).

Of these factors, the ROC analysis suggested that SUVmax (continuous) demonstrated good discriminative performance for both pT2 vs. pT1 (n=81, AUC: $0.866,95 \%$ CI: 0.790 to $0.942, \mathrm{P}<0.0001$, Figure $3 A$ ) and for pT2-3 vs. pT1 ( $\mathrm{n}=140$, AUC: $0.877,95 \%$ CI: 0.811 to 0.943 , $\mathrm{P}<0.0001$, Figure $3 B$ ). Youden's index was used to determine the cutoff as 7.4 for SUVmax (categorized) in all patients to fit clinical applications. The discriminating outcomes are presented in Figure 1. 
Page 6 of 12

Lin et al. Tumor SUVmax discriminates invasion depth

Table 3 Baseline risk factors for pT2-3 identified by univariate and multivariate logistic regression

\begin{tabular}{|c|c|c|c|c|c|c|}
\hline Characteristics & \multicolumn{3}{|c|}{ Univariate } & \multicolumn{3}{|c|}{ Multivariate } \\
\hline \multicolumn{7}{|l|}{ Age } \\
\hline$\leq 65$ (ref.) & Ref. & & & Ref. & & \\
\hline$>65$ & 2.321 & $1.118-4.819$ & 0.024 & 3.642 & $1.201-11.046$ & $0.022^{*}$ \\
\hline Female & Ref. & & & & & \\
\hline Male & 1.463 & $0.600-3.569$ & 0.403 & & & \\
\hline \multicolumn{7}{|l|}{ Smoking history } \\
\hline Non-smoking & Ref. & & & & & \\
\hline Non-drinking & Ref. & & & & & \\
\hline Drinking & 1.803 & $0.812-4.003$ & 0.147 & & & \\
\hline \multicolumn{7}{|l|}{ Differentiation of biopsy sample } \\
\hline bG1, 2, x (well, moderately) & Ref. & & & Ref. & & \\
\hline bG3 (poorly) & 2.698 & $1.067-6.825$ & 0.036 & 4.584 & $1.264-16.621$ & $0.021^{*}$ \\
\hline Unknown & 0.370 & $0.120-1.141$ & 0.084 & 0.124 & $0.018-0.865$ & $0.035^{\star}$ \\
\hline \multicolumn{7}{|l|}{ SUVmax of the primary tumors } \\
\hline Continuous, by 0.1 & 1.423 & $1.267-1.599$ & $<0.0001$ & 1.447 & $1.277-1.640$ & $<0.0001^{\star}$ \\
\hline
\end{tabular}

*, statistically significant. OR, odds ratio; $95 \% \mathrm{Cl}, 95 \%$ confidence interval; bG, biopsy differentiation grade; SUVmax, maximal standard uptake value.
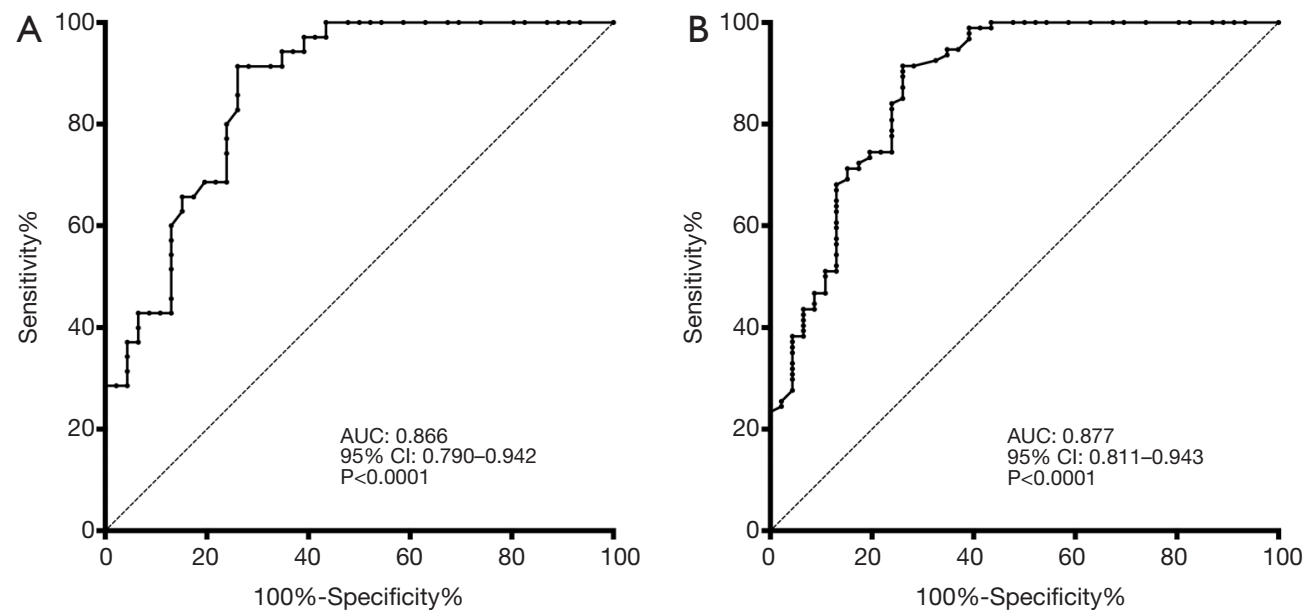

Figure 3 The discriminating performance of SUVmax (continuous) for invasion depth: SUVmax (continuous) had a good discriminating performance for (A) pT2 vs. pT1 (AUC: 0.866, 95\% CI: 0.7900 to 0.9423) and for (B) pT2-3 vs. pT1 (AUC: 0.877, 95\% CI: 0.811 to 0.943). SUVmax, maximal standard uptake value; AUC, area under the curve; 95\% CI, 95\% confidence interval. 
Table 4 The predictive model derived from independent baseline risk factors for pT2-3 vs. pT1

\begin{tabular}{|c|c|c|c|}
\hline Characteristics & \multicolumn{3}{|c|}{ Multivariate } \\
\hline \multicolumn{4}{|l|}{ Age } \\
\hline$\leq 65$ (ref.) & Ref. & & \\
\hline$>65$ & 3.288 & $1.129-9.574$ & $0.029^{*}$ \\
\hline bG1, 2 (well or moderately) & Ref. & & \\
\hline bG3 (poorly) & 3.913 & $1.043-14.676$ & $0.043^{*}$ \\
\hline Unknown & 0.324 & $0.074-1.426$ & 0.136 \\
\hline \multicolumn{4}{|l|}{ SUVmax } \\
\hline
\end{tabular}
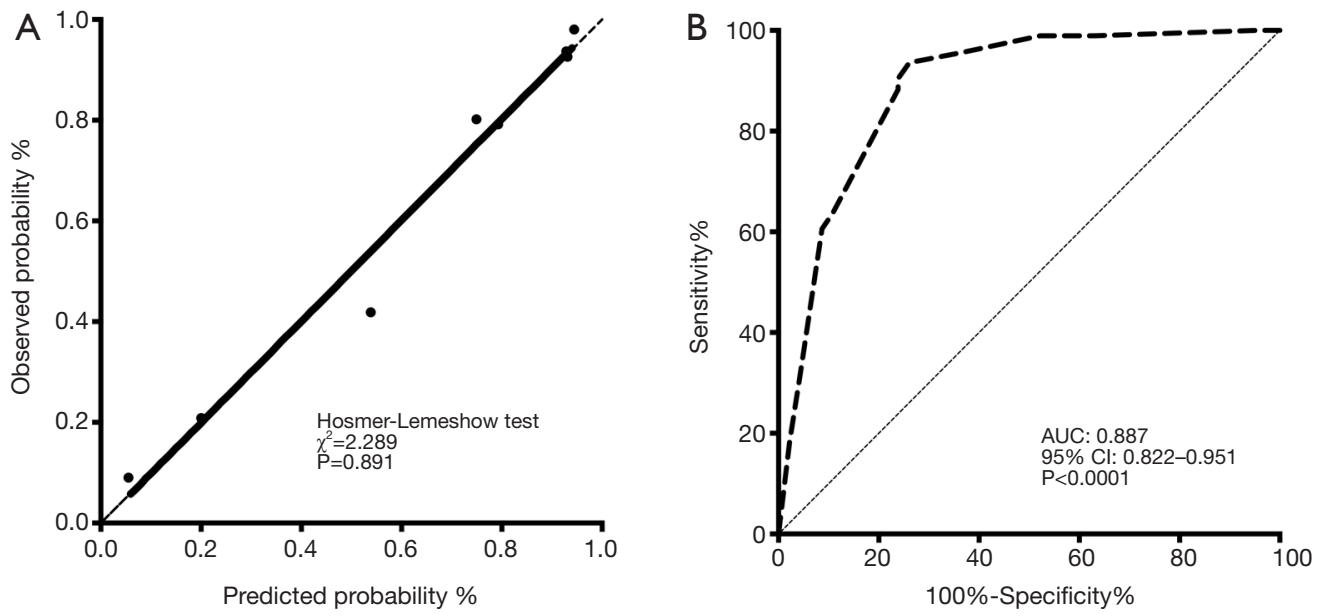

Figure 4 The discriminating performance of the novel model for pT2-3 vs. pT1. (A) The Hosmer-Lemeshow test indicated a good calibration (Chi-square 2.289, $\mathrm{P}=0.891$ ) and (B) the receiver operating curve indicated a high discrimination (AUC: $0.887,95 \% \mathrm{CI}: 0.822$ to 0.951) of the discriminating model. AUC, area under the curve; $95 \% \mathrm{CI}, 95 \%$ confidence interval.

\section{The discriminating model for $p T 2-3$}

According to the identification of baseline risk factors, the age categories ( $\leq 65 v s .>65)$, biopsy differentiation categories (well or moderately $v s$. poorly $v s$. unknown), and the primary tumor SUVmax categories ( $\leq 7.4 v s .>7.4)$ were included in the logistic regression analysis (enter method) to construct a novel model. As shown in Table 4, this model demonstrated good performance: accuracy $87.1 \%$, sensitivity $93.6 \%$, specificity $73.9 \%$, positive predictive value (PPV) $88.0 \%$, and negative predictive value (NPV) $85.0 \%$. The Hosmer-Lemeshow test indicated a good calibration (Chi-square 2.289, $\mathrm{P}=0.891$, Figure $4 A$ ), and the ROC analysis indicated high discrimination (AUC: $0.887,95 \%$ CI: 0.822 to $0.951, \mathrm{P}<0.0001$, Figure $4 B$ ) of the new model. The distribution of pT2-3 vs. pT1 in the 

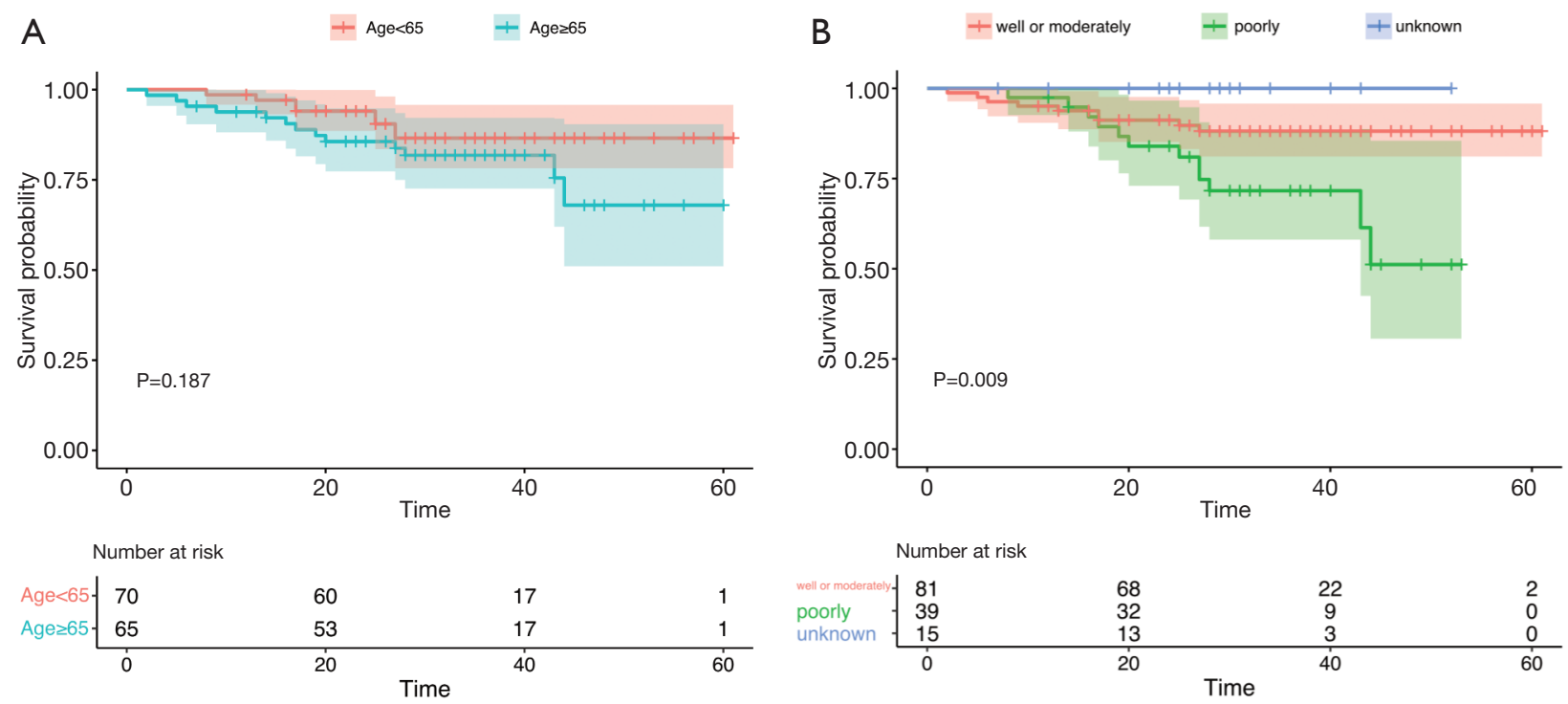

C
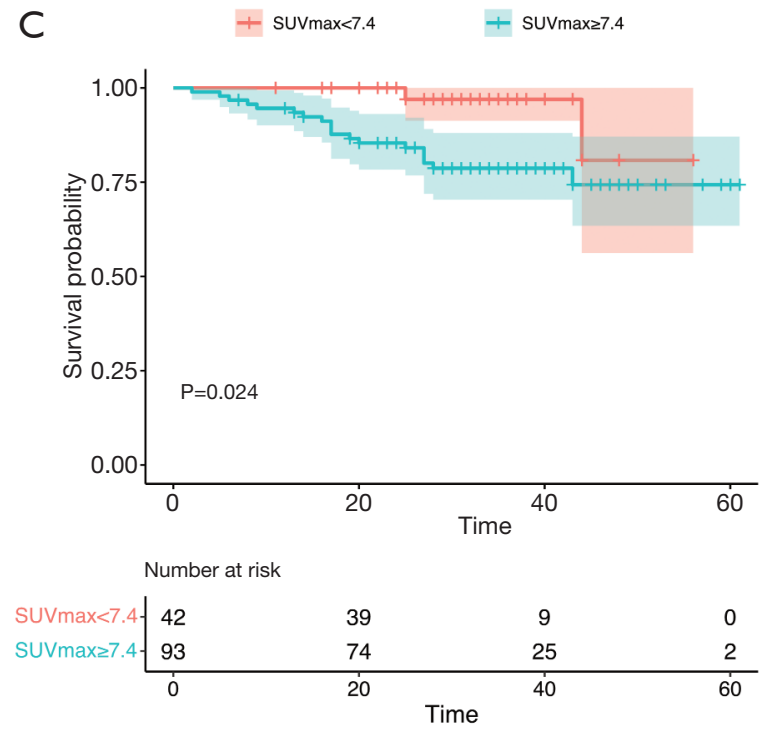

Figure 5 The survival curves of the risk factors for invasion depth: (A) age $<65 v s$. $\geq 65, \mathrm{P}=0.187$; (B) non-poorly differentiated $v s$. poorly differentiated, $\mathrm{P}=0.009$; and (C) SUVmax $<7.4$ vs. $\geq 7.4, \mathrm{P}=0.024$. SUVmax, maximal standard uptake value.

stratification of the model is shown in Table S2.

\section{Prognostic value of the baseline risk factors}

We then investigated whether the baseline risk factors were associated with overall survival. After excluding 5 patients lost to follow-up, 135 patients were included in the survival analysis. The median follow-up period was 35 months, and the 3 -year overall survival rate was $84.3 \%(78.0 \%$ to $91.1 \%)$. We found that age ( $\mathrm{P}=0.187$, Figure $5 A$ ) showed no significant difference, while biopsy differentiation $(\mathrm{P}=0.009$,
Figure $5 B)$ and primary tumor $\mathrm{SUV} \max (\mathrm{P}=0.024$, Figure 5C) showed significant differences in overall survival.

\section{Discussion}

The heated debate regarding cT2N0 esophageal cancer ultimately stems from the current clinical staging model's considerable inaccuracy. Pech et al. reported that the accuracy of cT1, cT2, and cT3 staged by endoscopic ultrasound (EUS) was $92 \%, 37 \%$, and $68 \%$, respectively (28). Dhupar et al. reported that the accuracy 
of cT1a, cT1b, cT2, and cT3 staged by EUS was $56 \%$, $58 \%, 10 \%$, and $70 \%$, respectively (29). Apart from EUS, $\mathrm{CT}$, which relies on wall thickness, cannot provide adequate information (30-32). Therefore, further study in this field would be on shaky ground as long as this problem remains unsolved. Because most of the cT2 category's migration lies in cT2 to pT1, finding effective methods to discriminate muscularis propria invasion is of great importance.

Consistent with previous studies (17-26), an increasing trend of primary tumor SUVmax was observed in the advanced pT categories. In this circumstance, the core question was whether this increasing trend could be translated to discriminate invasion depth prospectively. Huang et al. previously reported a positive result in a small study $(\mathrm{n}=45)$, where it showed great discriminative performance for $\mathrm{p} T$ categories $(\geq \mathrm{pT} 1$, AUC: 1.00 ; $\geq \mathrm{pT}$, AUC: 0.88; and $\geq$ pT3, AUC: 0.95) (17). However, in our study, we found SUVmax differed significantly in paired $\mathrm{pT}$ categories but not pT2 vs. pT3. Consequently, we found SUVmax (continuous) had good discrimination for $\mathrm{pT} 2-3$ vs. pT1, and pT2 vs. pT1, but not for pT3 vs. pT1-2 (AUC: $0.730,95 \%$ CI: 0.648 to 0.812 ). The discrepancy between studies might be attributed to institutional variations and selection bias. However, in general, the current published studies have similar outcomes regarding the difference in metabolic uptake between pT1 and their counterparts (9,17-19,31).

Although SUVmax (continuous) demonstrated good performance, we determined its optimal cutoff to fit clinical applications. The subsequent logistic regression model comprised of SUVmax (categorized), age, and biopsy differentiation achieved better discriminative performance. To our knowledge, this is the first comprehensive baseline model aimed at discriminating invasion depth. Furthermore, the model elements are more objective and less variable compared to EUS, which is technically demanding. Although endoscopic resection or dissection is useful to identify the mucosal or submucosal status, it cannot evaluate muscularis propria invasion due to the risk of severe perforation and delayed treatment. In our opinion, this novel model warrants consideration in future clinical trials.

With regards to the other pathological features, however, the published findings are not consistent. Some studies have reported that primary tumor SUVmax was correlated significantly with node metastasis, tumor differentiation, lymphatic invasion, and perineural invasion (17-20). However, other groups have reported contradictory outcomes (24-26). In our study, although invasion depth (pT2-3 vs. pT1) was correlated with SUVmax (Spearman, $\mathrm{P}<0.0001$ ), and with $\mathrm{pN}, \mathrm{pG}$, and LNI categories (Spearman, $\mathrm{P}=0.016, \mathrm{P}=0.001, \mathrm{P}<0.001$, respectively), SUVmax was not significantly correlated with $\mathrm{pN}, \mathrm{pG}$, and LNI categories. However, regarding the correlation between SUVmax and pathological staging (Spearman, $\mathrm{P}=0.008$ ), we believed that it was the correlation between SUVmax and invasion depth (pT2-3 vs. pT1) that worked as a bridge.

To our study, the sharp question is what is the foundation of this bridge, considering the limited spatial resolution of ${ }^{18} \mathrm{~F}-\mathrm{FDG}$ PET. This is currently not well understood. However, in a previous study in small-sized lung adenocarcinoma, evidence has demonstrated that SUVmax differed significantly between different histological subtypes and correlated with node metastasis (33). Although several studies have reported the differences of SUVmax in invasion depth (9,17-19,31), the biological mechanism remains to be elucidated. We posit that in early-stage cancer, the metabolic activity can provide certain information ahead of the visualization of spatial changes on current imaging modalities.

Previous studies have found that the current modalities are inadequate to determine the cT2 category $(9,10,28,29,34,35)$. Thus, EUS is not mandatory at our institution despite the guideline recommendations (3-5). It is often absent in real-world studies with large cohorts (8), including clinical trials (36). This is one limitation of our study. However, the satisfactory performance of SUVmax (continuous) and our newly proposed model highlight a promising role for metabolic activity in discriminating invasion depth.

There were some other limitations in our study. Firstly, this was a retrospective study with inherent selection bias. However, all patients received baseline PET-CT in our institution, which ensured a consistent protocol and interpretation. Secondly, some preoperative characteristics, such as endoscopic tumor diameter or length, were not included. However, the endoscopic measurement can have some errors in early-stage esophageal cancer. Moreover, in our study, 15 patients had no grading information, which would have certain effects on the model. Finally, although this model showed a satisfactory performance, it was not able to further discriminate against the invasion of muscularis propria (pT2) and adventitia (pT3), though cT3 tumors can often be identified by current modalities (37). This study is, therefore, a step toward addressing the heated debate on cT2N0 esophageal cancer. A prospective trial 
led by a multidisciplinary team is necessary to validate the findings and guide clinical practice.

\section{Conclusions}

Our novel baseline model comprised of age, biopsy differentiation grades, and primary tumor SUVmax provides a considerable discriminative performance in determining whether or not the muscularis propria is invaded. Further studies are necessary to validate the findings and guide clinical practice for cT2N0 esophageal cancer.

\section{Acknowledgments}

We sincerely acknowledge Zhengyang Hu for the help in statistical analyses, and C. Betlazar-Maseh and J. Chapnick from AME Editing Service for their help in language editing.

Funding: This study was supported by The Institutional Funding of Zhongshan Hospital Fudan University (No. 2019ZSFZ16, No. 2016ZSLC15). The funding had no influence on the design and interpretation of the study.

\section{Footnote}

Reporting Checklist: The authors have completed the STARD reporting checklist. Available at http://dx.doi.org/10.21037/ atm-20-4430

Data Sharing Statement: Available at http://dx.doi. org/10.21037/atm-20-4430

Conflicts of Interest: All authors have completed the ICMJE uniform disclosure form (available at http://dx.doi. org/10.21037/atm-20-4430). Dr. HW serves as an unpaid editorial board member of Annals of Translational Medicine from Jul 2019 to Jun 2021. The other authors have no conflicts of interest to declare.

Ethical Statement: The authors are accountable for all aspects of the work in ensuring that questions related to the accuracy or integrity of any part of the work are appropriately investigated and resolved. This study had been approved by the Institutional Review Board of Zhongshan Hospital Fudan University (HGBB-202006001) and was conducted in accordance with the Declaration of Helsinki (as revised in 2013). The informed consent was waived as the nature of retrospective studies.
Open Access Statement: This is an Open Access article distributed in accordance with the Creative Commons Attribution-NonCommercial-NoDerivs 4.0 International License (CC BY-NC-ND 4.0), which permits the noncommercial replication and distribution of the article with the strict proviso that no changes or edits are made and the original work is properly cited (including links to both the formal publication through the relevant DOI and the license). See: https://creativecommons.org/licenses/by-nc-nd/4.0/.

\section{References}

1. Bray F, Ferlay J, Soerjomataram I, et al. Global cancer statistics 2018: GLOBOCAN estimates of incidence and mortality worldwide for 36 cancers in 185 countries. CA Cancer J Clin 2018;68:394-424.

2. Napier KJ, Scheerer M, Misra S. Esophageal cancer: a review of epidemiology, pathogenesis, staging workup and treatment modalities. World J Gastrointest Oncol 2014;6:112-20.

3. Kuwano H, Nishimura Y, Oyama T, et al. Guidelines for diagnosis and treatment of carcinoma of the esophagus April 2012 edited by the Japan Esophageal Society. Esophagus 2015;12:1-30.

4. Lordick F, Mariette C, Haustermans K, et al. Oesophageal cancer: ESMO Clinical Practice Guidelines for diagnosis, treatment and follow-up. Ann Oncol 2016;27:v50-7.

5. Ajani JA, D'Amico TA, Bentrem DJ, et al. Esophageal and esophagogastric junction cancers, version 2.2019, NCCN Clinical Practice Guidelines in Oncology. J Natl Compr Canc Netw 2019;17:855-83.

6. D'Journo XB, Thomas PA. Current management of esophageal cancer. J Thorac Dis 2014;6 Suppl 2:S253-64.

7. Crabtree TD, Kosinski AS, Puri V, et al. Evaluation of the reliability of clinical staging of T2 N0 esophageal cancer: a review of the Society of Thoracic Surgeons database. Ann Thorac Surg 2013;96:382-90.

8. Esophageal Cancer Study Group Participating Centers. Predictors of staging accuracy, pathologic nodal involvement, and overall survival for cT2N0 carcinoma of the esophagus. J Thorac Cardiovasc Surg 2018. [Epub ahead of print].

9. Nelson DB, Mitchell KG, Weston BR, et al. Should endoscopic mucosal resection be attempted for cT2N0 esophageal cancer? Dis Esophagus 2019;32:1-6.

10. Luu C, Amaral M, Klapman J, et al. Endoscopic ultrasound staging for early esophageal cancer: are we denying patients neoadjuvant chemo-radiation? World J 
Gastroenterol 2017;23:8193-9.

11. Semenkovich TR, Panni RZ, Hudson JL, et al. Comparative effectiveness of upfront esophagectomy versus induction chemoradiation in clinical stage T2N0 esophageal cancer: a decision analysis. J Thorac Cardiovasc Surg 2018;155:2221-30.e1.

12. Samson P, Puri V, Robinson C, et al. Clinical T2N0 esophageal cancer: identifying pretreatment characteristics associated with pathologic upstaging and the potential role for induction therapy. Ann Thorac Surg 2016;101:2102-11.

13. Speicher PJ, Ganapathi AM, Englum BR, et al. Induction therapy does not improve survival for clinical stage T2N0 esophageal cancer. J Thorac Oncol 2014;9:1195-201.

14. Rice TW, Ishwaran H, Ferguson MK, et al. Cancer of the esophagus and esophagogastric junction: an eighth edition staging primer. J Thorac Oncol 2017;12:36-42.

15. Japan Esophageal Society. Japanese Classification of Esophageal Cancer, 11th Edition: part I. Esophagus 2017;14:1-36.

16. Kim TJ, Kim HY, Lee KW, et al. Multimodality assessment of esophageal cancer: preoperative staging and monitoring of response to therapy. Radiographics 2009;29:403-21.

17. Huang YC, Lu HI, Huang SC, et al. FDG PET using SUVmax for preoperative T-staging of esophageal squamous cell carcinoma with and without neoadjuvant chemoradiotherapy. BMC Med Imaging 2017;17:1.

18. Kato H, Kuwano H, Nakajima M, et al. Comparison between positron emission tomography and computed tomography in the use of the assessment of esophageal carcinoma. Cancer 2002;94:921-8.

19. Lim CH, Park YJ, Shin M, et al. Tumor SUVs on 18F-FDG PET/CT and aggressive pathological features in esophageal squamous cell carcinoma. Clin Nucl Med 2020;45:e128-33.

20. Miyawaki Y, Sato H, Fujiwara N, et al. Association of the primary tumor's SUVmax with survival after surgery for clinical stage IA esophageal cancer: a single-center retrospective study. Int J Clin Oncol 2020;25:561-9.

21. Kita Y, Okumura H, Uchikado Y, et al. Clinical significance of ${ }^{18} \mathrm{~F}$-fluorodeoxyglucose positron emission tomography in superficial esophageal squamous cell carcinoma. Ann Surg Oncol 2013;20:1646-52.

22. Sun G, Tian J, Gorospe EC, et al. Utility of baseline positron emission tomography with computed tomography for predicting endoscopic resectability and survival outcomes in patients with early esophageal adenocarcinoma. J Gastroenterol Hepatol 2013;28:975-81.
23. Kaida H, Kawahara A, Hayakawa M, et al. The difference in relationship between 18F-FDG uptake and clinicopathological factors on thyroid, esophageal, and lung cancers. Nucl Med Commun 2014;35:36-43.

24. Sun M, Li B, Fu Z, et al. Relationship between (18) F-fluorodeoxyglucose uptake in primary lesions and clinicopathological characteristics of esophageal squamous cell carcinoma patients. Exp Ther Med 2013;5:170-4.

25. Jiang W, Yang J, Lin X, et al. 18F-FDG PET-CT metabolic findings can predict the short-term curative effects in esophageal cancer. Int J Clin Exp Pathol 2019;12:4130-6.

26. Shimizu D, Yuasa N, Miyake H, et al. Clinical significance of SUVmax on preoperative $18 \mathrm{~F}$-fluorodeoxyglucose positron emission tomography in patients who underwent R0-esophagectomy for esophageal cancer. Nagoya J Med Sci 2018;80:401-9.

27. Fluss R, Faraggi D, Reiser B. Estimation of the Youden Index and its associated cutoff point. Biom J 2005;47:458-72.

28. Pech O, Günter E, Dusemund F, et al. Accuracy of endoscopic ultrasound in preoperative staging of esophageal cancer: results from a referral center for early esophageal cancer. Endoscopy 2010;42:456-61.

29. Dhupar R, Rice RD, Correa AM, et al. Endoscopic ultrasound estimates for tumor depth at the gastroesophageal junction are inaccurate: implications for the liberal use of endoscopic resection. Ann Thorac Surg 2015;100:1812-6.

30. Bunting D, Bracey T, Fox B, et al. Loco-regional staging accuracy in oesophageal cancer-How good are we in the modern era? Eur J Radiol 2017;97:71-5.

31. Jeong DY, Kim MY, Lee KS, et al. Surgically resected T1and T2-stage esophageal squamous cell carcinoma: $\mathrm{T}$ and $\mathrm{N}$ staging performance of EUS and PET/CT. Cancer Med 2018;7:3561-70.

32. Lowe VJ, Booya F, Fletcher JG, et al. Comparison of positron emission tomography, computed tomography, and endoscopic ultrasound in the initial staging of patients with esophageal cancer. Mol Imaging Biol 2005;7:422-30.

33. Nakamura H, Saji H, Shinmyo T, et al. Close association of IASLC/ATS/ERS lung adenocarcinoma subtypes with glucose-uptake in positron emission tomography. Lung Cancer 2015;87:28-33.

34. Hong SJ, Kim TJ, Nam KB, et al. New TNM staging system for esophageal cancer: what chest radiologists need to know. Radiographics 2014;34:1722-40.

35. Bergeron EJ, Lin J, Chang AC, et al. Endoscopic 
Page 12 of 12

ultrasound is inadequate to determine which T1/

T2 esophageal tumors are candidates for endoluminal therapies. J Thorac Cardiovasc Surg 2014;147:765-71; discussion 771-3.

36. Klevebro F, Alexandersson von Döbeln G, Wang N, et al. A randomized clinical trial of neoadjuvant chemotherapy versus neoadjuvant chemoradiotherapy for cancer of the

Cite this article as: Lin D, Liu G, Jiang D, Yu Y, Wang H, Shi H, Tan L. The role of primary tumor SUVmax in the diagnosis of invasion depth: a step toward clinical T2N0 esophageal cancer. Ann Transl Med 2021;9(2):112. doi: 10.21037/atm-20-4430

\section{Lin et al. Tumor SUVmax discriminates invasion depth}

oesophagus or gastro-oesophageal junction. Ann Oncol 2016;27:660-7.

37. Chao YK, Ku HY, Chen CY, et al. Induction therapy before surgery improves survival in patients with clinical T3N0 esophageal cancer: a nationwide study in Taiwan. Dis Esophagus 2017;30:1-7. 


\section{Supplementary}

Table S1 Lymph node metastasis in pathological T categories

\begin{tabular}{lccccc}
\hline Pathological T & pN0 & pN1 & pN2 & pN3 & pN+ \\
\hline pT1a & $10(90.9)$ & $1(9.1)$ & 0 & 0 & $1(9.1)$ \\
pT1b & $30(85.7)$ & $5(14.3)$ & 0 & 0 & $5(14.3)$ \\
pT2 & $27(77.1)$ & $4(11.4)$ & $3(8.6)$ & $1(2.9)$ & $8(22.9)$ \\
pT3 & $37(62.7)$ & $18(30.5)$ & $4(6.8)$ & 0 & $22(37.3)$ \\
\hline
\end{tabular}

*, Statistically significant; ${ }^{\S}, \mathrm{pT} 1 \mathrm{a}, 1 \mathrm{~b}, 2,3$ and pN0, pN+.

Table S2 The distribution of pT2-3 vs. pT1 in the stratification of the discriminating model

\begin{tabular}{|c|c|c|c|c|}
\hline SUVmax category & bG category & Age category & pT1, n (\%) & pT2-3, n (\%) \\
\hline$\leq 7.4$ & Well or moderately & $>65$ & $7(70.0)$ & $3(30.0)$ \\
\hline$\leq 7.4$ & Poorly & $\leq 65$ & $5(71.4)$ & $2(28.6)$ \\
\hline$\leq 7.4$ & Poorly & $>65$ & 0 & $2(100.0)$ \\
\hline$\leq 7.4$ & Unknown & $>65$ & $5(100.0)$ & 0 \\
\hline$>7.4$ & Well or moderately & $\leq 65$ & $6(20.7)$ & $23(79.3)$ \\
\hline$>7.4$ & Well or moderately & $>65$ & $2(6.9)$ & $27(93.1)$ \\
\hline$>7.4$ & Poorly & $\leq 65$ & $1(7.1)$ & $13(92.9)$ \\
\hline$>7.4$ & Unknown & $>65$ & $1(25.0)$ & $3(75.0)$ \\
\hline
\end{tabular}

SUVmax, maximal standard uptake value; bG category, biopsy differentiation grade category; pT, pathological T (invasion depth). 